\title{
SOBRE-MEDIDA EN EL LARGO DE TROZAS MÚLTIPLO DE Eucalyptus nitens PARA PRODUCIR CHAPAS DEBOBINADAS CALIDAD CLEAR
}

\author{
Dunn, Fernando ${ }^{1}$; Valencia, Juan Carlos $^{2}$; Soto, Luis ${ }^{3}$ y López, Cristián ${ }^{4}$
}

\section{RESUMEN}

Las crecientes restricciones a la cosecha de bosques nativos tropicales y templados generarán un déficit mundial de oferta de maderas duras para aserrío y tableros, abriendo una oportunidad de mercado para plantaciones de especies latifoliadas, entre las cuales destacan las especies del género Eucalyptus. A nivel nacional se suma la menor oferta proyectada de pino radiata.

Países con tradición en el cultivo de eucaliptos para producir fibra, como Brasil, Argentina, Uruguay, Australia, Sudáfrica y Chile, llevan varios años investigando, e incluso en algunos casos aplicando operacionalmente la silvicultura e industrialización de eucaliptos plantados para uso sólido. No obstante, uno de los principales obstáculos para el escalamiento comercial ha sido la existencia de elevados niveles de tensiones de crecimiento en la madera, que se manifiestan como rajaduras en los extremos de las trozas luego de la cosecha y que se acentúan en los procesos posteriores de madereo, transporte y macerado. Esto reduce el rendimiento de productos finales y afecta la eficiencia de la conversión industrial, tanto en el aserrío como en la industria de chapas y tableros.

Las rajaduras de punta de las trozas han sido un factor especialmente restrictivo en el caso chileno, en sus intentos de industrializar plantaciones podadas de la especie Eucalyptus nitens, y en particular para la producción de chapas debobinadas para cara de tableros contrachapados, por sus elevadas exigencias respecto a la tolerancia de rajaduras, lo que implica despuntar las trozas previo al debobinado, con la consiguiente pérdida de madera podada.

Teóricamente, una alternativa para mitigar este problema sería enviar desde el bosque trozas de largo múltiplo (TLM) para su trozado inmediatamente antes del debobinado, sin embargo se requiere conocer cuál es la medida de sobre largo (MSL) necesaria para asegurar el abastecimiento de trozas para torno con niveles de rajaduras aceptables, y a su vez conocer la pérdida en volumen que esto significa.

Para conocer cuál debería ser la MSL en TLM, en el año 2012 Forestal Mininco S.A. implementó estudios en temporadas de verano e invierno utilizando una muestra representativa de árboles podados desde un rodal de 19 años de edad, a partir de los cuales se obtuvo un total de 57 TLM podadas de $6,5 \mathrm{~m}$, las cuales fueron transportadas, maceradas, y posteriormente trozadas en largo debobinable $(2,6 \mathrm{~m})$ y despuntadas hasta lograr un nivel de rajaduras máximo definido por un índice $\operatorname{CSIR}<=8$, que se definió como el máximo para producir chapas para caras.

Como resultados, se obtuvo los valores de MSL para ambas temporadas, las magnitudes de despuntes en extremos basal y apical de las TLM, el nivel de pérdidas volumétricas y aprovechamiento, la evolución del índice CSIR durante las etapas del proceso y las relaciones entre las variables longitud de despunte y diámetro de trozas y longitud de trozas e índice CSIR. Se identificó además una buena correlación entre el índice CSIR y el índice Máximo Ancho de la Rajadura (MAR), el cual, previa validación futura, permitiría facilitar la segregación de rollizos usando el MAR.

\footnotetext{
${ }^{1}$ Forestal Mininco S.A, Chile, fernando.dunn@forestal.cmpc.cl

${ }^{2}$ Forestal Mininco S.A., Chile, jvalenciab@forestal.cmpc.cl

${ }^{3}$ Forestal Mininco S.A., Chile, Luis.Soto@forestal.cmpc.cl

4 clopezlan@vtr.net
} 
Se recomienda un rápido proceso de cosecha-transformación, enviar trozas en largos múltiplo con sobre-medida en el largo y un proceso industrial en línea que abarque descortezado, macerado, despunte y trozado de las TLM y debobinado.

Palabras clave: Eucalyptus nitens, chapas clear, rajaduras de punta de trozas, largo múltiplo.

\section{SUMMARY}

Growing restrictions on timber harvest of tropical and temperate natural forest are determining an increased supply shortage of high quality hardwood logs for sawn timber and veneer production. As a consequence, there is a market opportunity for plantation grown hardwood species such as Eucalyptus genus.

Producer countries of Eucalypts plantations for pulpwood production, such as Brazil, Uruguay, Argentina, Australia and Chile, have been researching and also industrializing some of their resource for solid-wood production. Although the occurrence and severity of high levels of growth stress at standing trees, related to the occurrence of log-end splitting after harvest, are particularly relevant in young Eucalypt plantation trees, limiting bigger progress at commercial scale as high levels of log end splits negatively impact on products recovery and processing efficiency.

Growth stress has been particularly restrictive for commercially producing high quality rotary peeler face grade veneer using pruned logs of Eucalyptus nitens cultivated in Chile. High quality face veneer allows only low levels of end splits being necessary to cut the log end with severe splits before peeling to satisfy the split allowance. As log end splitting are frequent in both logs ends in E. nitens, an alternative to mitigate this problem would be harvest, transport and steam treatment of multiple-length logs (TLM) and then cross cut them into $2.6 \mathrm{~m}$ peeler billets. However TLM must to include a magnitude of over-length (MSL) to allow cutting the excessive log end split sections immediately after steam treatment and peeling.

In order to know the MSL necessary to be added to the TLM to produce almost two peeler logs of $2.6 \mathrm{~m}$ long into a single TLM, in 2012 Forestal Mininco S.A. undertaken a to stage study summer and winter - on a 19 year old thinned and pruned $E$. nitens stand in which a total of 57 TLM of $6.5 \mathrm{~m}$ long each were harvested to be used as a sample. The 57 TLM were transported and steam treated in a plywood mill, and cross cut in their log-ends when the measured log end split index CSIR was higher than 8, immediately before to be cross cut and processed as $2.6 \mathrm{~m}$ peeler logs. The docked log end split section were measured in order to calculate the MSL for each TLM, and related to TLM log end diameter and CSIR.

The steam treatment of peeler log significantly increased the severity of long end splits in comparison to log end splits measured after harvest, being important to optimize the steam treatment schedule for $E$. nitens to minimize excessive losses due to log-end cross cut. Quick TLM deliveries from forest to plywood mill, including into the TLM a MSL, together an improved steam treatment schedules will surely increase the commercial processing viability of plantation-grown $E$. nitens for veneer production in Chile.

Key words: Eucalyptus nitens, face grade veneer, log end splitting, multiple log-length. 


\section{INTRODUCCIÓN}

Las crecientes restricciones a la cosecha de bosques nativos tropicales y templados generarán un déficit mundial de oferta de maderas duras para aserrío y tableros, abriendo una oportunidad de mercado para plantaciones de especies latifoliadas, entre las cuales destacan las especies del género Eucalyptus.

Países con tradición en el cultivo de eucaliptos para producir fibra, como Brasil, Argentina, Uruguay, Australia, Sudáfrica y Chile, llevan varios años investigando, e incluso en algunos casos aplicando operacionalmente la silvicultura e industrialización de eucaliptos plantados para uso sólido. No obstante, uno de los principales obstáculos para el escalamiento comercial, ha sido la existencia de elevados niveles de tensiones de crecimiento en la madera que se manifiestan como rajaduras en los extremos de las trozas luego de la cosecha y que se acentúan en los procesos posteriores de madereo, transporte y macerado (Yang and Waugh, 2001). Esto reduce el rendimiento de productos finales y afecta la eficiencia de la conversión industrial, tanto en el aserrío como en la industria de la chapa y tableros (Valencia et al., 2011).

Las rajaduras de punta de las trozas han sido un factor especialmente restrictivo en el caso chileno, en sus intentos de industrializar plantaciones podadas de la especie Eucalyptus nitens y, en particular, para la producción de chapas debobinadas para cara de tableros contrachapados, por sus elevadas exigencias respecto a la tolerancia de rajaduras, lo que implica despuntar las trozas previo al debobinado, con la consiguiente pérdida de madera podada (Meneses, 2011).

Teóricamente, una alternativa para mitigar este problema sería enviar desde el bosque trozas de largo múltiplo (TLM) para su trozado inmediatamente antes del debobinado, sin embargo se requiere conocer cuál es la medida de sobre largo (MSL) necesaria para asegurar el abastecimiento de trozas para torno con niveles de rajaduras aceptables y, a su vez, conocer la pérdida que esto significa en el valor.

Teniendo en cuenta experiencias anteriores en el sentido de que los trozos son más sensibles a presentar rajaduras en sus extremos cuando se cosechan en verano que en invierno, se realizaron dos estudios, uno con trozos múltiplos cosechados en marzo y el otro en agosto de 2012.

\section{OBJETIVOS}

Determinar la magnitud del sobre largo (MSL) que es necesario otorgar a trozas podadas de $E$. nitens, producto de la incidencia de las rajaduras de punta de troza, para obtener chapas de debobinado aptas para caras de tableros contrachapados.

Determinar el porcentaje de aprovechamiento volumétrico de las trozas podadas en la producción de chapas aptas para caras de tableros.

Determinar el nivel de avance de las rajaduras de los trozos de largo múltiplo (TLM) en distintas etapas del proceso industrial.

Las variables principales a cuantificar en este estudio son el largo individual y sumado de las secciones del extremo basal y apical de cada troza, que se deben eliminar antes del proceso de debobinado, en función de un nivel determinado de rajaduras que se considera aceptable para el proceso/producto a generar (índice CSIR máximo 8 ) $^{5}$

\footnotetext{
5 Índice CSIR, desarrollado en Sudáfrica para eucaliptos, expresa la severidad de las rajaduras, incluyendo en su cálculo la ponderación del número, largo y ancho de las rajaduras existentes, a mayor índice mayor severidad (Meneses 2011).
} 
El presente estudio reporta los resultados del sobre largo para un total de 37 TLM tomadas como muestra de temporada de verano y 20 TLM de invierno, todas de $6,5 \mathrm{~m}$ de largo cada una, cosechadas y procesadas en marzo y agosto de 2012, respectivamente.

\section{MATERIAL Y MÉTODO}

\section{Selección y Cosecha de Árboles y Generación de Trozas Múltiplo (TLM)}

Para la obtención de la muestra se eligió un rodal de Eucalyptus nitens de 19 años de edad, creciendo sobre suelo de trumaos de la precordillera andina, ubicado en el fundo Rucamanqui, Comuna de Tucapel, Provincia de Ñuble, Región del Bio Bio. La semilla que dio origen a este bosque, procedió de la zona de Toorongo Plateau, Victoria Central, Australia.

El rodal seleccionado fue podado y raleado oportunamente con esquemas aptos para la generación de madera libre de nudos. A la fecha de obtención de las muestras habían aproximadamente 400 árboles/ha, con poda hasta $6,5 \mathrm{~m}$ de altura, aproximadamente.

Los árboles se seleccionaron a modo de raleo. Se eligieron 40 para la muestra de verano, cubriendo diámetros de TLM de $24 \mathrm{~cm}$ y más. Estos se redujeron a 20 en el caso de la muestra de invierno, basados en experiencia del estudio previo, respecto de la falta de relación entre largo de despunte y diámetro de trozos. A todos los árboles seleccionados se les midió y registró el DAP. De esta manera se obtuvo 40 y $20 \mathrm{TLM}$, respectivamente, de 6,5 m de largo.

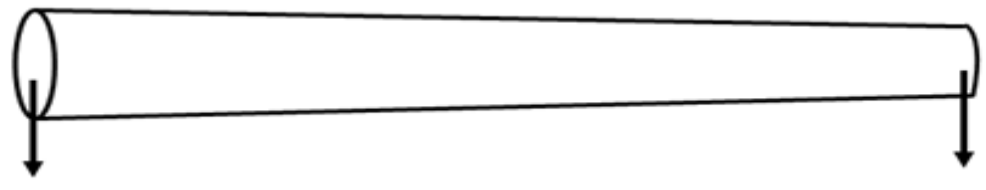

Cara basal (1)

Cara apical (2)

Figura $\mathrm{N}^{0} 1$

TROZA LARGO MÚLTIPLO (TLM)

Se consideró dicha longitud de TLM con el propósito de que en el mejor de los casos, sin necesidad de despunte, permitiera obtener para debobinado: 2 trozos de 2,6 m (long grain) y un trozo de 1,3 m de largo (short grain). En su defecto, obtener al menos 2 trozas de 2,6 $\mathrm{m}$ y una sobre-medida de 1,3 $\mathrm{m}$ para despunte, considerando la suma de ambas caras, basal y apical de la TLM (Figura N 1).

En los Cuadros $\mathrm{N}^{\circ} 1$ y $\mathrm{N}^{\circ} 2$ se muestra los datos descriptivos de los árboles seleccionados y de los trozos múltiplos obtenidos de estos.

Tanto el volteo como el trozado fueron realizados con motosierra (Figura $\mathrm{N}^{\circ} 2$ ). Posterior al trozado, cada TLM fue debidamente identificada (número de árbol e identificación de la base y ápice). Posteriormente las TLM fueron trasladadas con bueyes hasta orilla de camino (Figura $\mathrm{N}^{\circ} 2$ ), lugar donde se procedió al descortezado en forma manual (Figura $\mathrm{N}^{\circ} 3$ ).

Luego se aplicó sellante con brocha en los extremos con el objeto de aminorar el ritmo de pérdida de humedad y por consiguiente, minimizar severidad de rajaduras en extremos. Al mismo 
tiempo se midieron diámetros, el CSIR y el máximo ancho de rajadura (MAR) en extremos basal y apical de todas las TLM. A esto se denominó Momento 1 de medición (M1).

Cuadro $\mathrm{N}^{\circ} 1$

DESCRIPCIÓN ÁRBOLES SELECCIONADOS Y TLM

OBTENIDAS COMO MUESTRA DE VERANO

\begin{tabular}{|l|c|c|c|c|}
\hline Variable & Media & Mínimo & Máximo & DS \\
\hline DAP árboles $(\mathrm{cm})$ & 37,0 & 29,5 & 44,1 & 4,4 \\
\hline $\mathrm{N}$ \%árboles y trozas & 40 & & & \\
\hline Largo troza $(\mathrm{m})$ & 6,5 & 6,5 & 6,5 & 0 \\
\hline LED troza $(\mathrm{cm})$ & 37,8 & 31,3 & 45,4 & 4,1 \\
\hline SED troza $(\mathrm{cm})$ & 31,4 & 25,0 & 37,5 & 4,0 \\
\hline SED JAS troza $(\mathrm{cm})$ & 30 & 24 & 36 & 4,0 \\
\hline Volumen $\left(\mathrm{m}^{3} \mathrm{ssc}\right)$ & 0,641 & 0,400 & 0,876 & 0,161 \\
\hline
\end{tabular}

LED: Diámetro troza en su punta gruesa

SED: Diámetro de la troza en su punta delgada.

DS: Desviación Estándar

\section{Cuadro $\mathrm{N}^{\circ} 2$}

DESCRIPCIÓN ÁRBOLES SELECCIONADOS Y TLM OBTENIDOS COMO MUESTRA DE INVIERNO

\begin{tabular}{|l|c|c|c|c|}
\hline Variable & Media & Mínimo & Máximo & DS \\
\hline DAP árboles $(\mathrm{cm})$ & 39,8 & 35,3 & 47,1 & 3,6 \\
\hline LED troza $(\mathrm{cm})$ & 40,0 & 36,4 & 46,2 & 2,9 \\
\hline SED troza $(\mathrm{cm})$ & 33,5 & 30,2 & 38,7 & 2,7 \\
\hline SED JAS troza $(\mathrm{cm})$ & 32,7 & 30,0 & 38,0 & 2,6 \\
\hline Volumen $\left(\mathrm{m}^{3} \mathrm{ssc}\right)$ & 0,742 & 0,625 & 0,989 & 0,117 \\
\hline
\end{tabular}

LED: Diámetro troza en su punta gruesa

SED: Diámetro de la troza en su punta delgada.

DS: Desviación Estándar
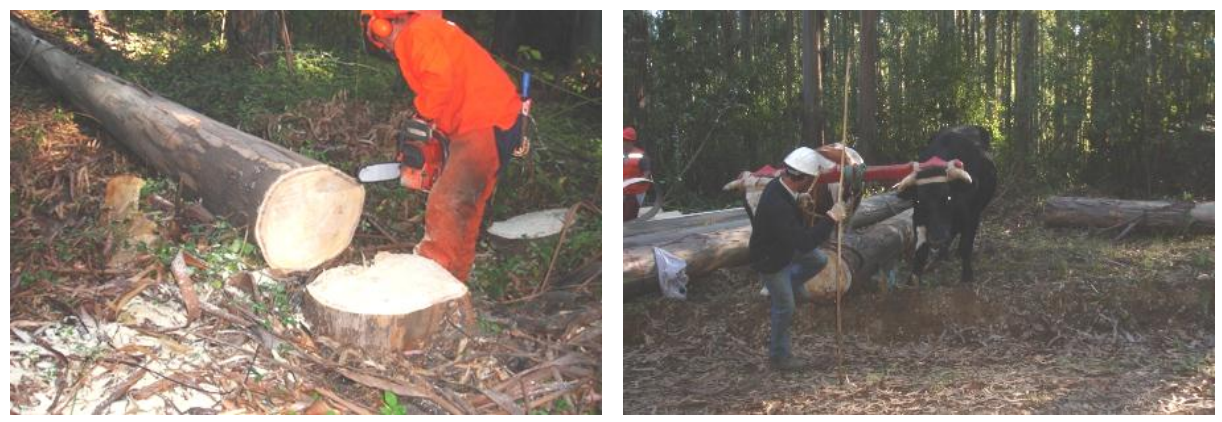

Figura $\mathrm{N}^{\circ} 2$

FAENAS EN BOSQUE. VOLTEO, TROZADO TLM Y MADEREO 
El transporte de las TLM al lugar del ensayo, Planta de Paneles Angol, se efectuó mediante camión con carro auto cargable, dentro de las 24 horas siguientes al volteo de los árboles en el caso del estudio de verano y dentro de las 12 horas siguientes a éste, en el caso del estudio de invierno.

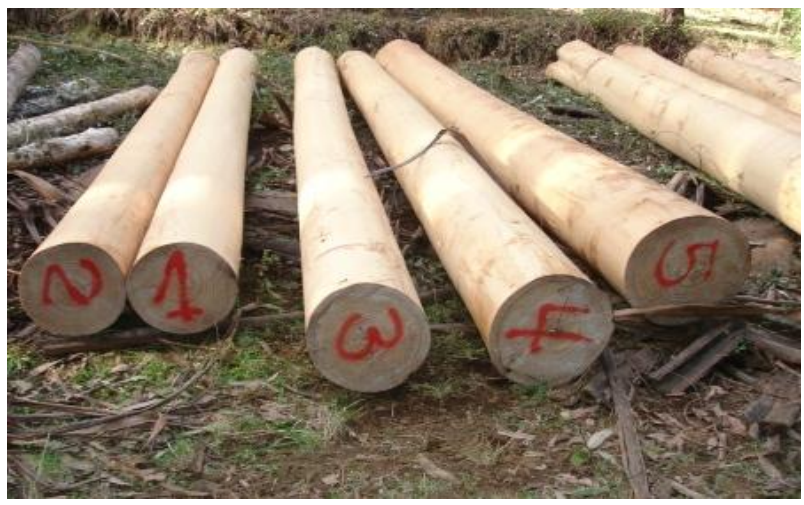

Figura $\mathbf{N}^{\circ} 3$

TLM DESCORTEZADOS E IDENTIFICADOS EN BOSQUE

En el caso del estudio de verano, una vez en la planta de debobinado de Paneles Angol, los trozos se descargaron a piso del patio, con un tractor trineumático. Luego y solo para la muestra de verano, se procedió a la medición y registro del CSIR y MAR pre-macerado (Momento 2 = M2). A continuación la misma máquina los puso sobre carros para ingresar a la cámara de macerado.

En el caso del estudio de invierno el M2 se saltó, en consideración a los resultados obtenidos en el estudio de verano, de manera que los TLM se descargaron del camión directamente sobre los carros para el macerado y prontamente fueron introducidos a la cámara.

\section{Macerado}

En el caso del estudio de verano, el macerado de las TLM se realizó según programa industrial de la planta de Paneles Angol, considerando 12 horas de macerado en vapor. Este se efectuó durante la noche y se detectaron deficiencias en el control del mismo.

En el caso del estudio de invierno se respetó un programa de macerado diseñado por el Jefe de Planta. Este consideró 10,5 h según el esquema de la Figura $\mathrm{N}^{\circ} 4$.

En cuanto se sacó los TLM de la cámara de secado, estos fueron tendidos sobre rollizos y tapados con una carpa para aminorar el ritmo de rajaduras durante el tiempo de despunte y trozado. Luego a medida que los sacaba para despunte y trozado, para la muestra de verano, se les medía el CSIR y MAR en ambos extremos (Momento $3=$ M3) (Figura N5).

Los primeros tres TLM sirvieron para probar el protocolo de despunte. La cantidad de TLM a despuntar y su volumen según clase diamétrica en el estudio de verano y de invierno fue de 37 y de 20 , respectivamente (Cuadros $\mathrm{N}^{\circ} 3$ y $\mathrm{N}^{\circ} 4$ ). 


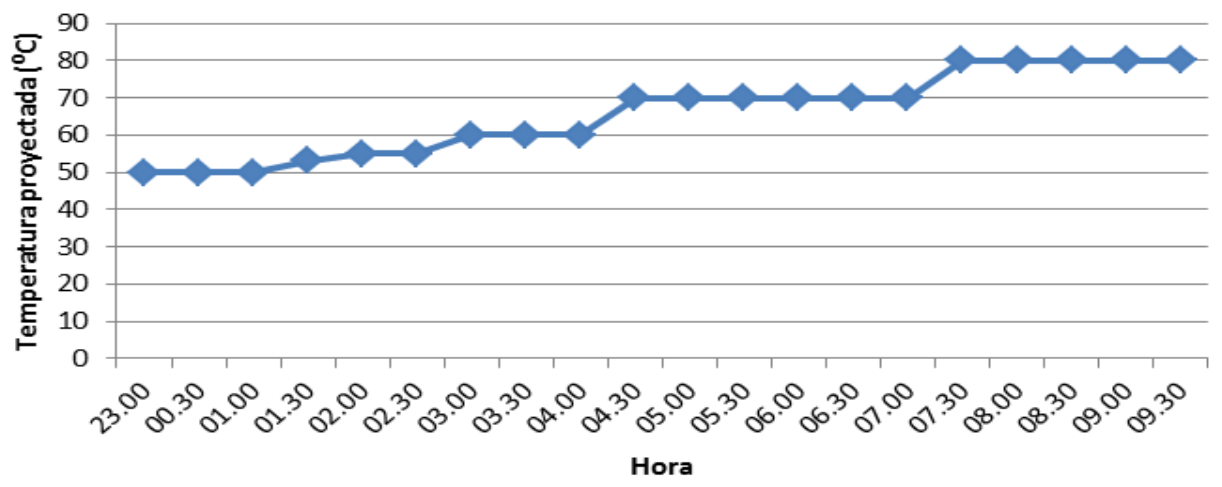

Figura $\mathrm{N}^{\circ} 4$

PROGRAMA DE MACERADO APLICADO

Cuadro $\mathrm{N}^{\circ} 3$

CANTIDAD DE TLM ESTUDIO VERANO Y VOLUMEN SEGÚN CLASE DIAMÉTRICA

\begin{tabular}{|c|c|c|}
\hline $\begin{array}{c}\text { SED } \\
(\mathbf{c m})\end{array}$ & $\begin{array}{c}\text { Trozas } \\
\left(\mathbf{N}^{\mathbf{}}\right)\end{array}$ & $\begin{array}{c}\text { Volumen JAS } \\
\left(\mathbf{m}^{\mathbf{3}} \mathbf{~ s s c}\right)\end{array}$ \\
\hline 24 & 5 & 2,00 \\
\hline 26 & 5 & 2,33 \\
\hline 28 & 5 & 2,69 \\
\hline 30 & 6 & 4,31 \\
\hline 32 & 4 & 3,49 \\
\hline 34 & 6 & 4,70 \\
\hline 36 & 6 & 6,13 \\
\hline Total & $\mathbf{3 7}$ & $\mathbf{2 5 , 6 5}$ \\
\hline
\end{tabular}

Cuadro $\mathrm{N}^{\circ} 4$

CANTIDAD DE TLM ESTUDIO INVIERNO Y VOLUMEN SEGÚN CLASE DIAMÉTRICA

\begin{tabular}{|c|c|c|}
\hline $\begin{array}{c}\text { SED } \\
(\mathbf{c m})\end{array}$ & $\begin{array}{c}\text { Trozas } \\
\left(\mathbf{N}^{\circ}\right)\end{array}$ & $\begin{array}{c}\text { Volumen JAS } \\
\left(\mathbf{m}^{\mathbf{3}} \mathbf{~ s s c}\right)\end{array}$ \\
\hline 30 & 7 & 4,373 \\
\hline 32 & 5 & 3,539 \\
\hline 34 & 3 & 2,389 \\
\hline 36 & 4 & 3,559 \\
\hline 38 & 1 & 0,989 \\
\hline Total & $\mathbf{2 0}$ & $\mathbf{1 4 , 8 4 9}$ \\
\hline
\end{tabular}

\section{Despunte y Trozado}

El despunte y trozado se efectuó con motosierra, empezando los cortes por el extremo basal de la TLM, cortando al final de la rajadura observada en la periferia de cada TLM, e identificando los despuntes (Figura $\mathrm{N}^{\circ}$ 5). Luego, se procedió al trozado a 2,6 m de largo, para 
generar una troza basal para torno. Si se evidenciaba rajadura mayor a CSIR 8 en la cara apical de la troza basal, se procedía al despunte hasta lograr dicho objetivo (Figura $\mathrm{N}^{\circ} 6$ ). Si el largo residual de la troza basal después del despunte era inferior o superior a 2,6 m, se procedía a eliminar la sección sobrante.

El mismo procedimiento anterior se siguió con la siguiente sección de $2,6 \mathrm{~m}$, para generar una troza apical de dicho largo.
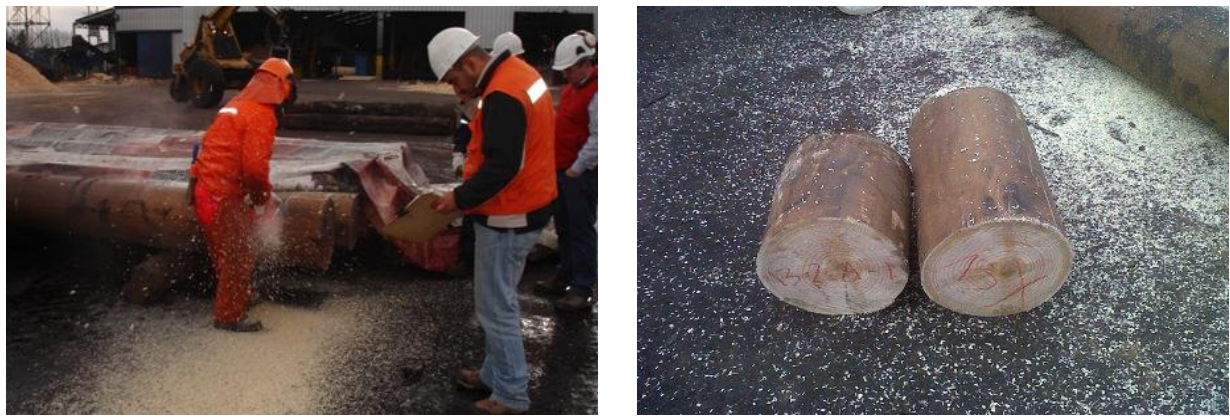

Figura $\mathrm{N}^{\circ} 5$

DESPUNTE DE TROZAS E IDENTIFICACIÓN DE DESPUNTES

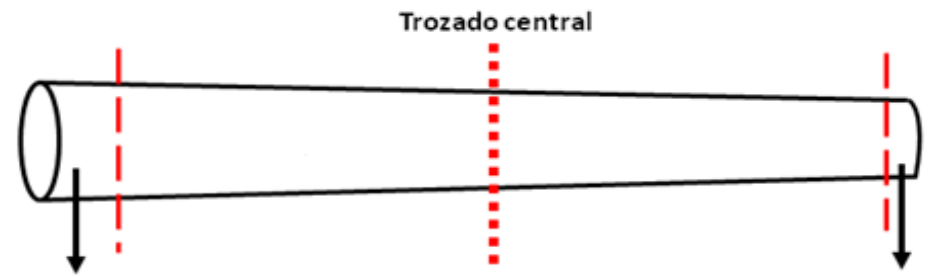

Despunte basal (B1)

B2 / A1

Despunte apical (A2)

B1= troza basal, cara basal

B2 = troza basal, cara apical

A1 = troza apical, cara basal

A2 = troza apical, cara apical.

Figura $N^{\circ} 6$

TROZA BASAL Y TROZA APICAL

En el caso de las TLM que no requirieron despunte, se procedió a confeccionar una tercera troza para torno de 1,3 m de largo desde la sección apical de la TLM. Se midió y registró: el largo y diámetro de las trozas basales y apicales finales y de las secciones descartadas, para posteriormente proceder a las cubicaciones respectivas.

Cabe señalar que una troza de la muestra de invierno se dejó fuera de los análisis por arrojar un valor muy fuera de rango. 


\section{RESULTADOS}

\section{Magnitud del Sobrelargo de Trozas múltiplos TLM}

En el $100 \%$ de las TLM muestras de verano fue necesario efectuar algún despunte. Un $89 \%$ de ellas fueron despuntadas en su base y un $97 \%$ en su ápice. En el centro de la troza no fue necesario despuntar, pues los cortes no presentaron rajaduras con CSIR mayor a 8.

En la muestra de invierno, en un $70 \%$ de las TLM fue necesario el despunte. Del total de esta muestra, un $60 \%$ requirió despunte basal y un $55 \%$ despunte apical. En el centro de la TLM fue necesario despuntar 2 trozas, lo cual equivalió a un $10 \%$ de la muestra (Figura $\mathrm{N}^{\circ} 7$ ). En la misma figura se aprecia que en la muestra de verano hubo que despuntar el $89 \%$ de las TLM en su base en comparación con el $60 \%$ de las de invierno, y en su ápice hubo que despuntar un $97 \%$ de la muestra de verano y un $55 \%$ de las de invierno.

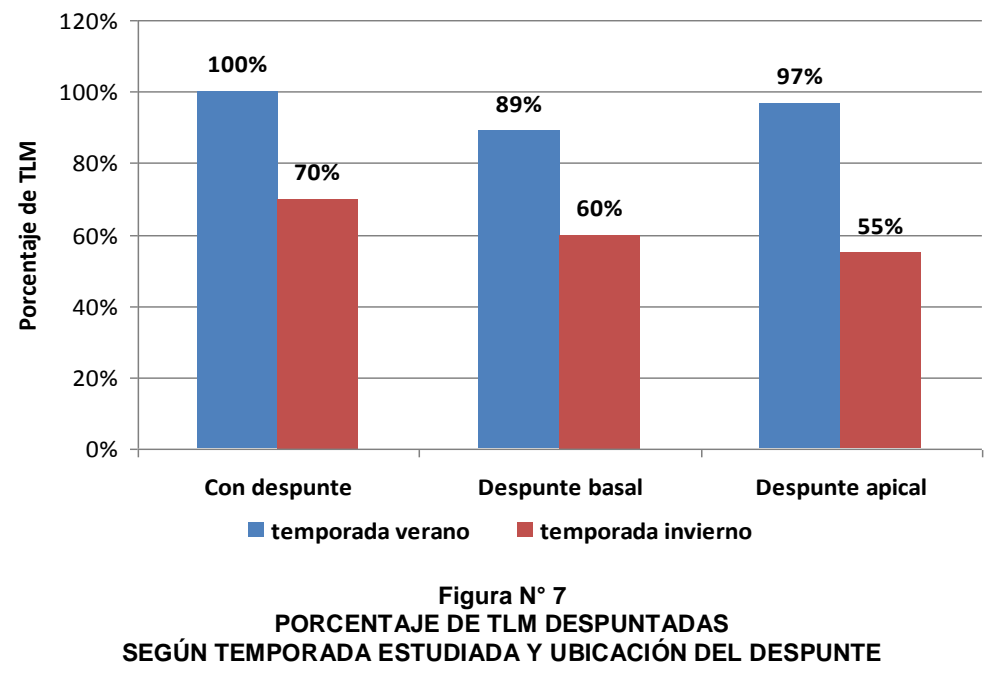

Las cifras señaladas confirman que en cuanto a cantidad de TLM afectadas por rajaduras en sus extremos es bastante mayor el problema de rajaduras de las trozas de temporada de verano que de invierno. Sin embargo, en el centro de estas el problema es menor, resultando en los ensayos realizados solo secciones centrales de TLM de invierno (10\%) con índice CSIR mayor a 8. Esto se atribuye a excesivas tensiones en algunas trozas, condición que podría ocurrir en cualquiera época del año con esta especie.

Cabe señalar que se puede aminorar las rajaduras del centro de la troza por la vía de un proceso de trozado industrial en línea, tendiente a minimizar el tiempo de exposición de las trozas a la intemperie. Además, se debe tener en cuenta que el proceso del estudio de invierno fue más corto y mejor controlado, lo cual debe haber contribuido a lograr mejores resultados en esta temporada.

\section{Sección Basal de Trozas Múltiplo TLM}

En la Figura $\mathrm{N}^{\circ} 8$ se observa que mientras en temporada de invierno, un $40 \%$ de las TLM no sufrieron despunte en su sección basal, en las de verano estas fueron solo el $11 \%$. La longitud 
promedio de los despuntes basales de las TLM en que fue necesario despuntar alcanzó a $34 \mathrm{~cm}$ en la temporada de verano y a $29 \mathrm{~cm}$ en la de invierno. El rango que estos alcanzaron fue de 13 a 95 $\mathrm{cm}$ en temporada de verano y de $7 \mathrm{a} 63 \mathrm{~cm}$ en invierno.

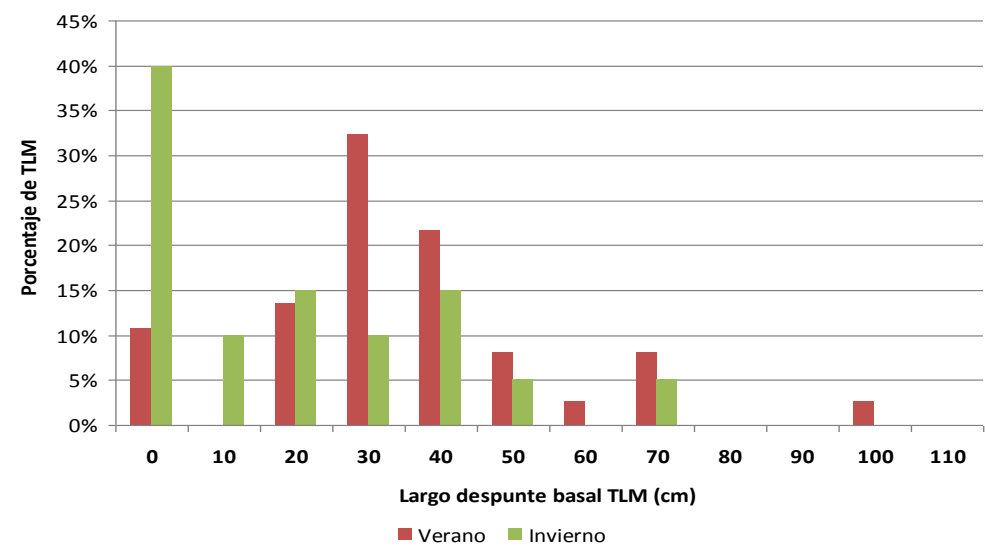

Figura $\mathrm{N}^{\circ} 8$

DISTRIBUCIÓN DE FRECUENCIA PORCENTUAL DE TLM EN SECCIÓN BASAL SEGÚN LARGO DE DESPUNTE APLICADO EN VERANO E INVIERNO

\section{Sección Apical de Trozas Múltiplo (TLM)}

En la Figura $\mathrm{N}^{\circ} 9$ se observa que mientras en la temporada de invierno un $45 \%$ de las TLM no necesitaron despunte en su sección apical, en la de verano solo un $3 \%$ no necesitó de esta práctica. La longitud promedio de los despuntes apicales de las TLM en que fue necesario despuntar alcanzó a $54 \mathrm{~cm}$ en temporada de verano y a $32 \mathrm{~cm}$ en temporada de invierno. El rango de estas fue de 21 a $139 \mathrm{~cm}$ en verano y de 20 a $61 \mathrm{~cm}$ en invierno.

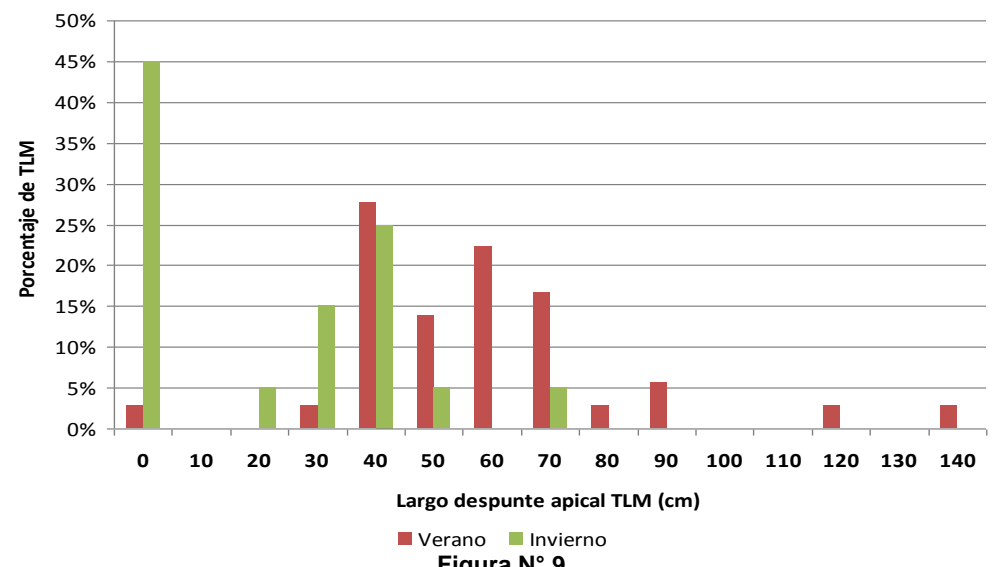

DISTRIBUCIÓN DE FRECUENCIA PORCENTUAL DE TLM EN SECCIÓN APICAL SEGÚN LARGO DE DESPUNTE APLICADO EN VERANO E INVIERNO 


\section{- Suma de Despuntes de Secciones Basal y Apical}

En verano, la suma de las longitudes de despunte basal y apical para las TLM que hubo que despuntar fue de $88 \mathrm{~cm}$ en promedio, mientras que en invierno esta cifra fue de $61 \mathrm{~cm}$, afectando en este último caso al $70 \%$ de las TLM.

En la Figura $\mathrm{N}^{\circ} 10$ se muestra las frecuencias parcial y acumulada del número de TLM según la longitud de despunte total considerando ambas secciones, basal y apical. Se aprecia la diferencia entre ambas temporadas, siendo la temporada de invierno significativamente menos severa en términos de la magnitud del despunte requerido para lograr un CSIR máximo de 8. Por ejemplo, en la temporada de invierno, el $35 \%$ de las TLM experimentó, a nivel individual, un largo de despunte superior a los $60 \mathrm{~cm}$, mientras que en la temporada de verano dicho porcentaje fue de un $70 \%$ de las TLM, vale decir el doble.

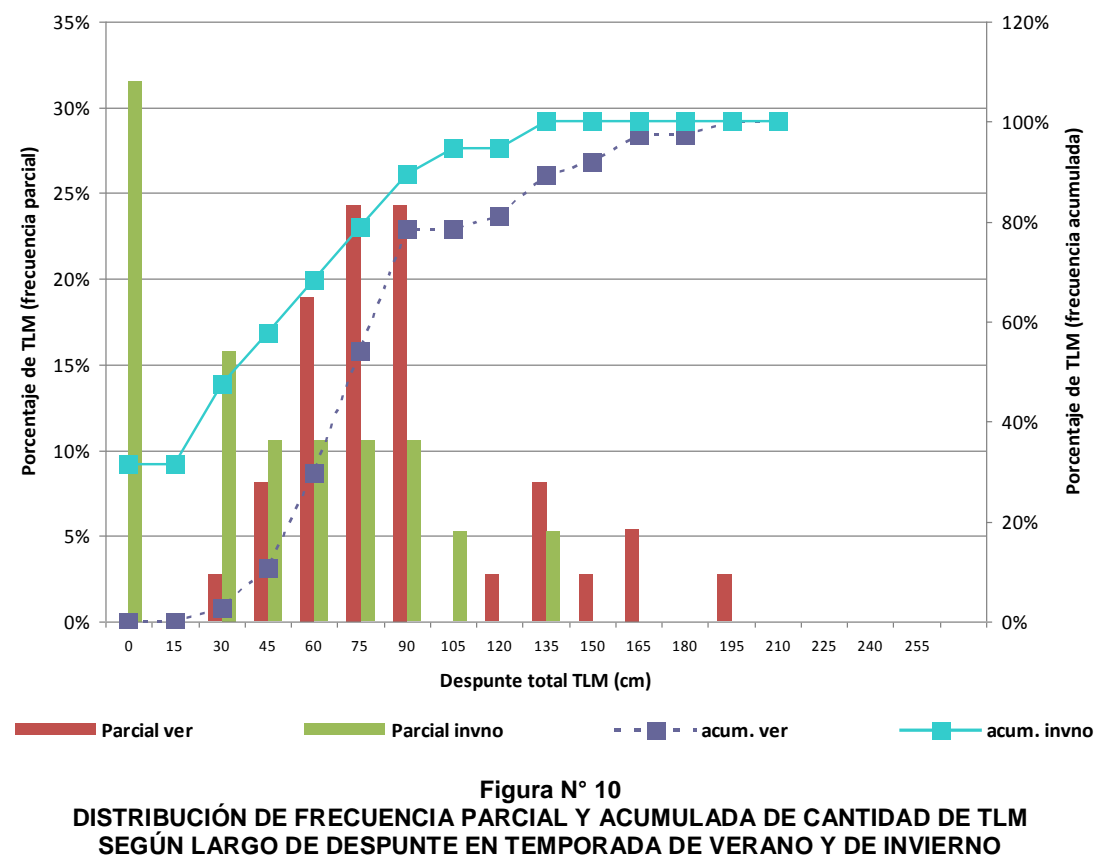

Se aprecia también en la figura que de las TLM aproximadamente un $80 \%$ en verano y un $85 \%$ en invierno necesitarían un sobrelargo no superior a $90 \mathrm{~cm}$ para obtener 2 trozas debobinables de 2,6 $\mathrm{m}$ de largo aptas para caras, de cada una de estas.

Si se consideran TLM podadas de $6,5 \mathrm{~m}$ de largo, de acuerdo a los resultados de la muestra de verano, del $86 \%$ de estas se generarían 2 trozas debobinables de 2,6 m de largo. En el caso de temporada de invierno, esta cifra ascendería a 90\%.

Todas las cifras antes señaladas indican que el abastecimiento con trozas múltiplo en invierno sería notablemente más favorable que en verano, pues permitiría operar con una menor sobredimensión en el largo de las TLM. Sin embargo, un proceso más rápido y más adecuado 
desde el bosque hasta el trozado en planta debería mejorar en forma importante los resultados en términos de la cantidad de trozas a despuntar y de la longitud de los despuntes, en especial en verano.

También se debe considerar que los despuntes basales, tanto en verano $(34 \mathrm{~cm}$ en promedio) como en invierno $(29 \mathrm{~cm})$, resultaron menores que los apicales $(54 \mathrm{~cm}$ en verano y 32 $\mathrm{cm}$ en invierno).

Este resultado es favorable bajo dos aspectos. La sección más gruesa a eliminar mediante el despunte es la más corta (menor volumen de pérdida) y la sobredimensión apical se puede manejar de manera que esta provenga de un tramo no podado del árbol, minimizando el valor de la pérdida, e incluso dándole una magnitud tal, que permita obtener una troza debobinable de calidad apta para chapas interiores de tableros.

\section{Pérdida Volumétrica por Despuntes}

El porcentaje promedio de pérdidas volumétricas por despunte de TLM podadas para láminas de caras fue de $13,2 \%$ (rango de $4-29 \%$ ) para temporada de verano y $8,4 \%$ (rango de 0 $21 \%$ ) para la de invierno. Esto equivale a que el $86,8 \%$ en verano y el $91,6 \%$ en invierno del volumen de la TLM de 6,5 $\mathrm{m}$ de largo podría ser aprovechado para debobinar.

En la Figura $\mathrm{N}^{\circ} 11$ se muestra la distribución de frecuencia de dichas pérdidas. Se observa por ejemplo que en temporada de verano un $78 \%$ de las TLM tuvieron entre 5 y $15 \%$ de volumen de despunte. En invierno en tanto el $90 \%$ de las TLM sufrió $15 \%$ y menos de volumen de despunte, incluso un $30 \%$ de ellas no sufrió pérdidas debido a éste.

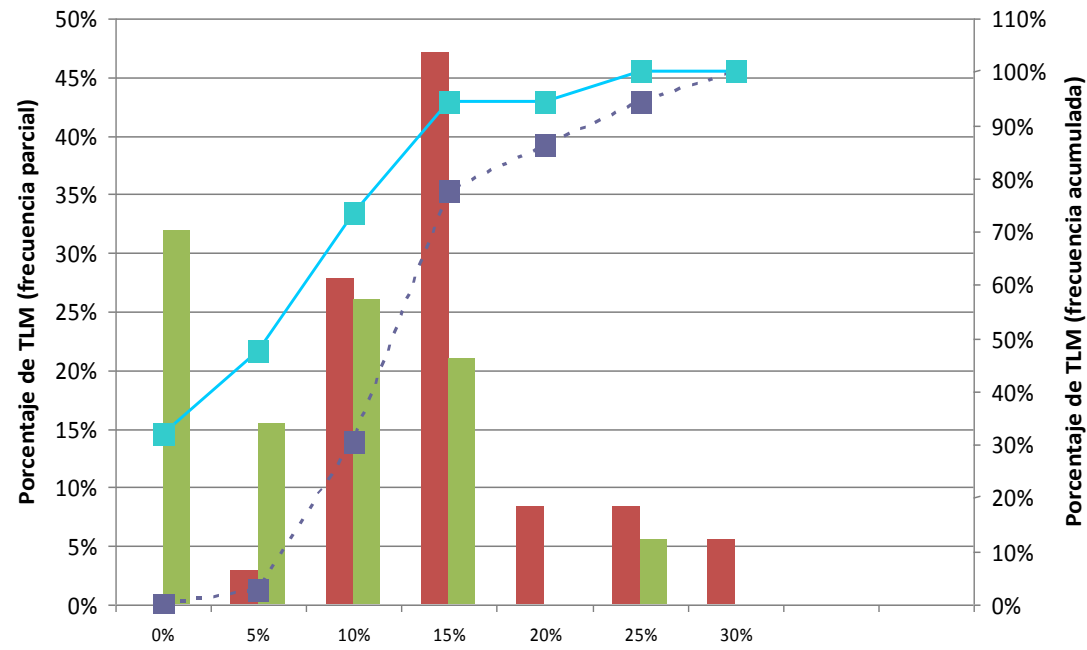

Proporción volumen de despunte total respecto al volumen total de la TLM

\footnotetext{
Parcial verano

Parcial invierno

- - - - acumulado verano

- acumulado invierno

Figura $\mathrm{N}^{\circ} 11$

DISTRIBUCIÓN DE FRECUENCIA PARCIAL Y ACUMULADA DE CANTIDAD DE TLM SEGÚN PORCENTAJE VOLUMEN DE DESPUNTE EN TEMPORADA DE VERANO Y DE INVIERNO
} 
Además de mediante las mejoras posibles y necesarias del proceso entre el bosque y el trozado en planta industrial, las pérdidas volumétricas podrían reducirse si se maneja el sobrelargo apical de la TLM, de manera que este afecte básicamente a la sección no podada de los árboles, permitiendo incluso que esta sea aprovechada en un 100\% para chapas de interior de tableros, aspecto que será materia de estudio futuro.

\section{Relación Diámetro de Trozas Múltiplo con la Magnitud del Despunte}

En la Figura $N^{\circ} 12$ se muestra la relación entre el diámetro menor (SED) y el largo total de despunte de la TLM, para la muestra de la temporada de verano y la de invierno. Se observa claramente que no hay relación entre dichas variables. Esto significa que la magnitud de la rajadura de los extremos de los trozas sobre CSIR 8, no está relacionada con el SED de estas. Cabe señalar que este mismo análisis se efectuó para el DAP de los árboles seleccionados y el diámetro mayor (basal) de las TLM, en relación al despunte, no encontrándose correlación entre ellas.
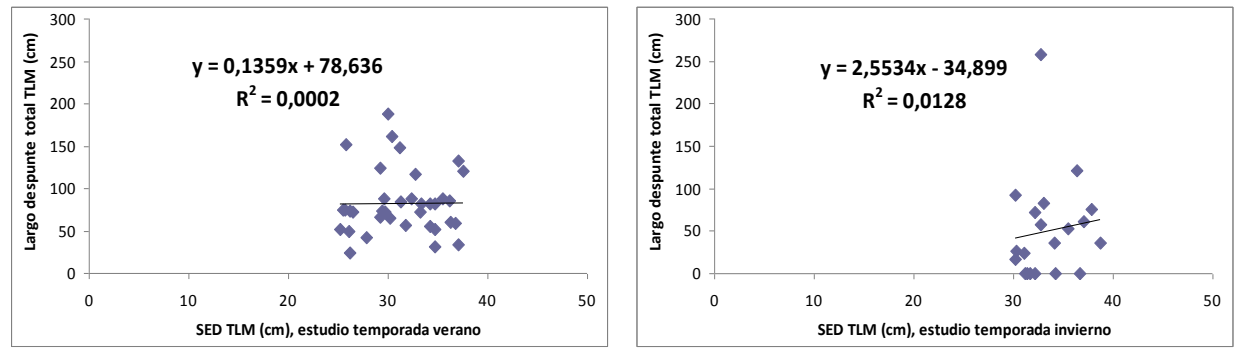

\section{Figura $N^{\circ} 12$ \\ RELACIÓN SED CON LONGITUD DE DESPUNTE MUESTRA DE TLM TEMPORADA DE VERANO Y DE INVIERNO}

\section{Evolución del Índice CSIR}

Con la muestra de verano se estudió la variación del índice CSIR en el tiempo en los extremos basal y apical de las TLM, vale decir en 3 momentos: M1: en bosque (pos trozado), M2: pre macerado en planta industrial y M3: pos macerado en planta industrial. En la Figura $\mathrm{N}^{\circ} 13 \mathrm{se}$ muestra los resultados de las mediciones de dicho índice. Se aprecia un notable aumento de las rajaduras en la medida que pasa el tiempo, en especial después del macerado.

Por otra parte, se aprecia que la cara apical de la TLM es la que más se afecta. Se recuerda que el proceso de macerado de esta muestra de trozas tuvo problemas de control, lo cual le agregó una condición indeseada a la primera muestra, en verano, situación que fue mejorada para la de invierno.

Cabe agregar que el $81 \%$ de las TLM registró un CSIR igual o inferior a 8 cuando fue medido en el bosque (M1). Este porcentaje disminuyó a un $62 \%$ en M2 (premacerado) y a un $8 \%$ en M3 (inmediatamente después del macerado), incluso al momento de realizar el despunte se redujo a $0 \%$, pues fue necesario despuntar todas las TLM por no cumplir con la restricción de CSIR 8 impuesta en este estudio para obtener trozas debobinables aptas para caras de tableros contrachapados.

Esto es un indicador claro de la importancia de un proceso rápido y bien controlado desde la cosecha hasta el debobinado para una especie tan sensible a rajarse en los extremos de los rollizos. 


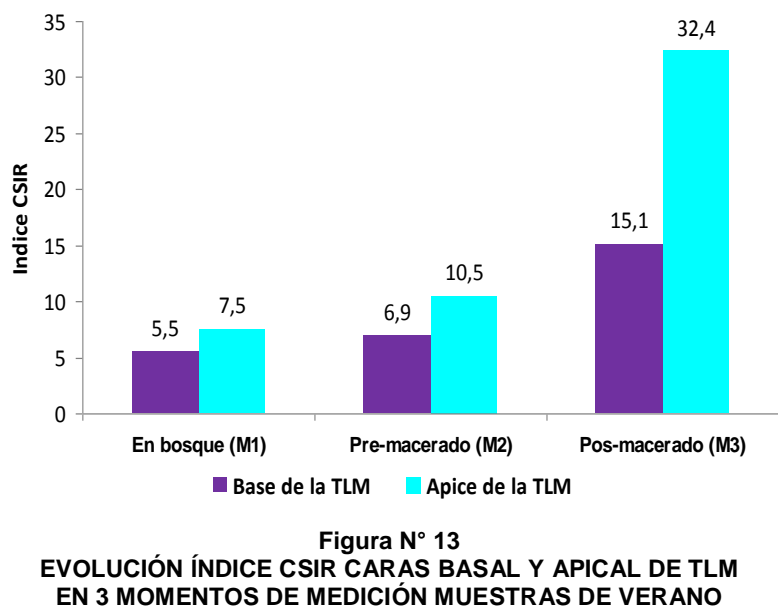

\section{Relación Índice CSIR e Índice MAR}

Adicionalmente a la medición del índice CSIR, en el presente trabajo se propuso estudiar como índice de rajadura alternativo el índice MAR. Este, medido después del macerado (M3), registró una excelente correlación con el CSIR, tal como demuestra la Figura $N^{\circ} 14$. Utilizando la regresión ajustada entre ambas variables, se determinó que un índice CSIR de 8 equivale a un MAR de $3 \mathrm{~mm}$, es decir TLM con un MAR de $3 \mathrm{~mm}$ o menor se pueden procesar para producir chapas para cara.

La ventaja del MAR respecto al CSIR es su facilidad de uso a escala operacional, por su rápida medición y comprensión, siendo una contribución relevante del presente estudio y que se recomienda continuar explorando en futuros trabajos con esta especie y otras.

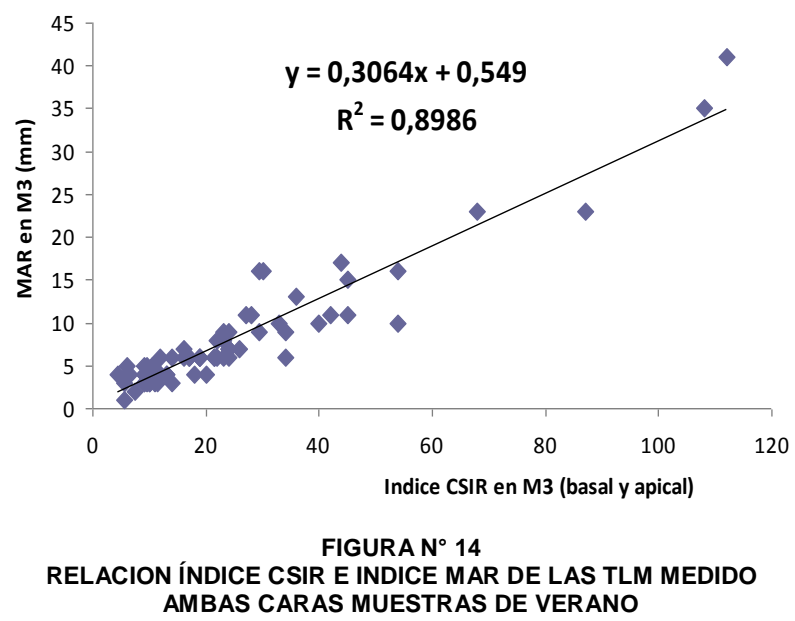




\section{CONCLUSIONES}

Se confirma la importancia de usar trozas de largo múltiplo (TLM) de Eucalyptus nitens y la necesidad de despuntar al menos la gran mayoría de estas (100\% en verano y $70 \%$ en invierno), con el fin de producir chapas para caras de tableros contrachapados en usos de apariencia.

En promedio, se necesitaría un sobre largo de $88 \mathrm{~cm}$ en verano y de $61 \mathrm{~cm}$ en invierno, para obtener dos trozas debobinables de 2,6 m, con el propósito señalado, a partir de una troza múltiplo de $6,5 \mathrm{~m}$ de largo. Esta sobredimensión permitiría obtener 2 trozas podadas del $86 \%$ de las TLM en verano y del $90 \%$ de estas en invierno. Si se desea que esta proporción sea mayor, se requeriría un mayor sobrelargo, debiendo ser este de hasta $2 \mathrm{~m}$ en verano y 1,30 m en invierno para garantizar que el $100 \%$ de las TLM proporcionen 2 trozas debobinables de 2,6 m.

La magnitud del despunte basal fue en promedio de $34 \mathrm{~cm}$ en verano y de $29 \mathrm{~cm}$ en invierno, resultando ser menor que el apical de las TLM, con valores de 54 y $32 \mathrm{~cm}$ en promedio, respectivamente. Además este fue mayor en la muestra de verano que en la de invierno.

En general no se requirió efectuar despuntes en las secciones centrales de las TLM, debido a que el debobinado se realizó inmediatamente después del trozado, lo que hace recomendable considerar un proceso en línea para el trozado de las TLM.

Las pérdidas de volumen por efecto de los despuntes fue en promedio de $13,2 \%$ en verano y de $8,4 \%$ en invierno. Esto equivale a un 87 y $92 \%$ de aprovechamiento volumétrico de las TLM en verano y en invierno, respectivamente.

Las rajaduras de punta de trozas aumentaron considerablemente desde la cosecha hasta el trozado pre-debobinado, variando el índice CSIR en promedio en la sección basal de las TLM de 5,5 a 15,5 entre la medición en bosque y la de después del macerado. Esto indica la necesidad de un proceso lo más rápido posible entre la cosecha y el debobinado.

El diámetro de las trozas no se correlacionó con la longitud de los despuntes.

Se obtuvo una excelente correlación entre el índice CSIR y el máximo ancho de rajadura (MAR), indicador que a futuro se recomienda validar, pues operacional presenta ventajas en cuanto a su simpleza y rapidez para segregar rollizos.

Los resultados obtenidos en este estudio permitirán identificar la necesidad de:

- $\quad$ Un proceso rápido y adecuado desde la cosecha hasta el debobinado.

- $\quad$ El uso de trozas de largo múltiplo (TLM), que incluyan en la sobre-medida, una fracción sin poda en el extremo apical de esta, para maximizar el aprovechamiento de la madera clear.

- Un proceso industrial en línea que incluya descortezado, macerado, despunte de TLM, trozado de estas a largo debobinable y debobinado inmediato.

\section{REFERENCIAS}

Meneses, M., 2011. Calidad trozas, aserrío y secado de E. nitens. Primer Seminario Regional INFOR, CORMA y PIT Nitens. Presentación. 44pp.

Valencia, J. C.; Harwood, C.; Washusen, R.; Morrow, A.; Wood, M.; Volker, P., 2011. Longitudinal growth strain as a log and wood quality predictor for plantation-grown Eucalyptus nitens sawlogs Wood Sci. Technol. 45 (1):15-34.

Yang, J.; Waugh, G., 2001. Growth stress, its measurement and effects. Australian Forestry 64(2):127-135. 
